\title{
Oralité et notations, de Chine en Europe
}

\section{François Picard}

\section{(2) OpenEdition \\ Journals}

Édition électronique

URL : http://journals.openedition.org/ethnomusicologie/674

ISSN : 2235-7688

Éditeur

ADEM - Ateliers d'ethnomusicologie

Édition imprimée

Date de publication : 1 janvier 1999

Pagination : 35-53

ISBN : 978-2-8257-0680-0

ISSN : 1662-372X

Référence électronique

François Picard, «Oralité et notations, de Chine en Europe », Cahiers d'ethnomusicologie [En ligne],

12 | 1999, mis en ligne le 08 janvier 2012, consulté le 19 avril 2019. URL : http://

journals.openedition.org/ethnomusicologie/674

Ce document a été généré automatiquement le 19 avril 2019.

Tous droits réservés 


\title{
Oralité et notations, de Chine en Europe
}

\author{
François Picard
}

1 J'ai ailleurs consacré un chapitre de synthèse à la présentation et à l'historique des notations chinoises (Picard 1991) ainsi qu'un article intitulé « Les notations musicales en Chine » (Picard 1999). Il ne s'agit donc plus ici d'exposer leur historique ni même leur fonctionnement, mais leur utilisation et ce que nous apprennent leurs avantages respectifs. Après une présentation des deux principales notations en vigueur en Chine, la notation ancienne en caractères chinois gongche, remplacée au $\mathrm{xx}^{\mathrm{e}}$ siècle par la notation chiffrée Chevé, je voudrais mettre en valeur deux de ses caractéristiques : son oralité et son indépendance vis-à-vis du système officiel (les cinq notes wusheng et les douze hauteurs absolues lülü). Ces deux caractéristiques font de la notation chiffrée Chevé un précieux outil pour une pédagogie actuelle et pour tant de musiciens réfractaires au solfège, vécu comme un outil de classe, contraignant, à l'accès réservé à une élite instruite, fait par et pour la musique classique, perçue comme bourgeoise.

2 A travers quelques exemples, en particulier un des plus fameux airs chinois, «La feuille de saule » (Liu ye jin), j'aimerais montrer que le passage d'une notation à une autre est une traduction. Notons au passage que l'usage avéré du terme « transcription » pour désigner l'inscription, la scription, d'une musique non écrite prévaut (verba valent usu) sur l'étymologie et retire certes au mot «transcription» (Amiot $1754: 128)$ sa valeur pour désigner ce que Mireille Helffer appelle "transnotation" mais que cette dernière opération serait mieux appelée tout simplement « traduction » (La Borde 1780 : 146).

\section{La notation gongche}

3 La notation gongche s'écrit, comme un texte chinois, de haut en bas en colonnes verticales alignées de droite à gauche. Sept caractères chinois, qui se prononcent respectivement shang-che-gong-fan-liu-wu-yi, notent le nom des sept notes correspondant à do-ré-mi-fasol-la-si. L'adjonction d'un graphème permet de noter les notes do, ré et mi de l'octave supérieure, tandis que les sol-la-si graves sont écrits avec trois caractères supplémentaires he-si-yi. Cette notation des hauteurs est relative, et la clé ou tonalité peut 
varier selon les genres, les instruments, les occasions. Elle peut être précisée en début ou en cours de partition par un emprunt à des nomenclatures qui pour le coup sont relativement variées. Le rythme est indiqué partiellement par les espaces laissées et, plus précisément, la mesure l'est par des signes (ronds vides ou pleins, apostrophes) en regard, indiquant la frappe de la claquette ou du tambour, c'est-à-dire la récurrence des groupes d'un, deux ou quatre temps, éventuellement plus. Tout ceci est bien connu et attesté. Amiot (1754) en a donné une première description, ainsi qu'une traduction sur portée.

Les notations en caractères chinois du type gongche ${ }^{1}$ sont de très loin les plus courantes en Chine du XI $\mathrm{x}^{\mathrm{e}}$ siècle au début du $\mathrm{xx}^{\mathrm{e}}$ et, contrairement à ce qui est encore écrit parfois en Occident, leur système de référence est heptatonique et non pentatonique. Une assertion sans fondement, mais largement répandue dans la littérature secondaire, expose en effet que « au XIV siècle [...] les envahisseurs mongols apportèrent avec eux une gamme et un système de notation différents de ceux utilisés par les Chinois » (Van Aalst 1884: 15). Cette affirmation a été reprise sans précaution par Georges Soulié (1911: 9), Louis Laloy (1912 : 58-59, 83), Jacques Pimpaneau (1988), Tràn Van Khê (1990 : 951). Soulignons que Perny (1872: 149), Maurice Courant (1913: 95-96, 157), puis, toujours en français, Ma Hiao-Tsiun (1976: 191), avaient cité les sources incontestables qui interdisent de colporter une telle contrevérité. Chinois (Jao 1971: 80), Japonais ou spécialistes américains contemporains (Chao 1969: IX) n'ont jamais commis une telle erreur. Au-delà de l'ensemble, considérable, des travaux de ces derniers, et parallèlement à la tâche de déchiffrement des partitions de Dunhuang, datées du $\mathrm{x}^{\mathrm{e}}$ siècle au plus tard et qui utilisent comme chacun s'accorde à le dire une notation de sept sons à l'octave, ma participation au Groupe de recherche Histoire des sciences et techniques en Asie orientale m'a fait lire le Mengqi bitan (Shen 1095) plus attentivement que je ne l'avais encore fait. Shen Gua (1031-1095) - donc avant Koubilaï -, outre sa mention bien connue de la notation gongche (Shen 1095 : tiao 531), rapporte (Shen 1095 : tiao 99) - ce qui à ma connaissance n'a jamais été relevé ni en Chine ni ailleurs - qu'à son époque on trouvait au Shanxi une inscription attribuée à un homme des Tang, "écrite en un genre de sanskrit ", dont on disait que c'était la notation d'un air bien connu².

\section{La notation chiffrée}

5 La notation chiffrée, inventée par Rousseau (1742) et introduite du Japon en Chine au début $\mathrm{du} \mathrm{xx} \mathrm{x}^{\mathrm{e}}$ siècle, s'écrit horizontalement de gauche à droite. Elle comprend les signes de hauteur 1234567, prononcés do-ré-mi-fa-sol-la-si. Les octaves inférieures et supérieures sont marquées par des points souscrits ou suscrits. La hauteur ainsi indiquée est relative à une note de référence prise dans un autre système. Les Chinois ont ici emprunté le système germano-anglais CDEFGAB pour désigner les hauteurs absolues. En cas de métabole ou de modulation, on peut à l'intérieur d'une même pièce changer de système de référence. On écrit $1=C$ pour indiquer qu'on est « en ton de do ». Le 0 (zéro, non prononcé) indique un silence dont la valeur de durée n'est pas en soi spécifiée. Il y a des barres de mesure, verticales. La valeur de durée est par défaut la noire. Un soulignement indique une croche, un double soulignement la double croche. On peut pointer les notes. Un trait horizontal suivant une note sur la même ligne indique le doublement de sa valeur. On peut altérer les notes par des dièses ou des bémols, les relier, et en fait ajouter toutes les marques secondaires - valeurs d'intensité, indications 
d'attaques, appoggiatures, signes de reprise, changements de tempo, variations de vitesse - pour en faire l'équivalent exact de la portée.

\section{Rousseau et la notation musicale chinoise}

6 Dès Du Halde (1735: T. III, 266), l'Europe put savoir que les Chinois possédaient une notation musicale. « Ils n'ont point comme nous des Notes de Musique, ni aucun signe qui marque la diversité des tons, les élévations ou les abaissements de la voix, \& toutes ces variations qui font l'harmonie. Ils ont néanmoins quelques caractères qui font connaître les divers tons. » Du Halde cependant emploie des termes suffisamment ambigus pour que quelques années plus tard Jean-Jacques Rousseau (1712-1778) (1768: 74) s'y trompe : «Caractères de musique » "Il n'y a que les Nations de l'Europe qui sachent écrire leur Musique. Quoique dans les autres parties du Monde chaque Peuple ait aussi la sienne, il ne paraît pas qu'aucun d'eux ait poussé les recherches jusqu'à des Caractères pour la noter. Au moins est-il sûr que les Arabes ni les Chinois, les deux Peuples étrangers qui ont le plus cultivé les Lettres, n'ont, ni l'un ni l'autre, de pareils Caractères. A la vérité les Persans donnent des noms de Villes de leurs pays ou des parties du corps humain aux quarantehuit Sons de leur Musique. Ils disent, par exemple, pour donner l'intonation d'un Air Allez de cette Ville à celle-là ; ou Allez du doigt au coude. Mais ils n'ont aucun signe propre pour exprimer, sur le papier, ces mêmes Sons. Et quand aux Chinois, on trouve dans le père Du Halde qu'ils furent étrangement surpris de voir les Jésuites noter et lire sur cette même Note tous les Airs chinois qu'on leur faisait entendre. " L'ambiguïté réside dans les mots «notes" et "caractères", mais elle est surtout dans un aveuglant sentiment ethnocentrique de suprématie du système européen. Rousseau en effet cite plus loin (1768 : 314) un « Air Chinois tiré du P. du Halde » (fig. 1).

Fig. 1: Notation sur portée, «La feuille de saule» (Du Halde 1735: I.328). Du Halde, comme plus tard parfois Amiot, note en clé de sol 1 ère ligne, ce qui causa des erreurs de lecture

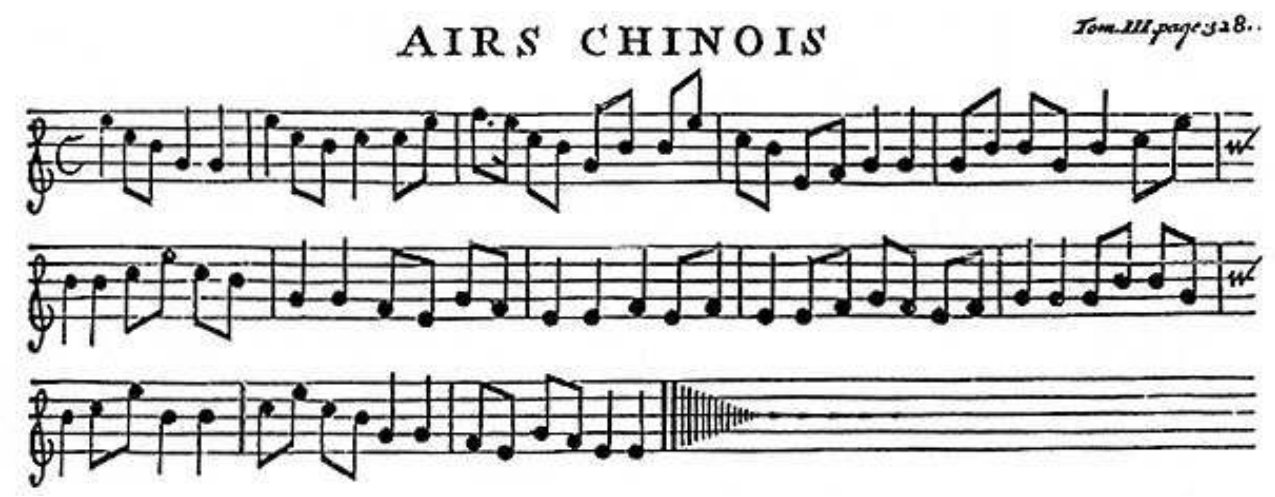

Or cette notation n'est que la traduction d'une notation chinoise que reproduit plus tard Amiot, qui ajoute $(1754: 135-136)$ : «Les notes et les autres signes que les Chinois emploient dans leur musique étant une fois connus et comparés à nos notes et à nos signes musicaux, il n'est plus difficile de noter leurs airs à notre manière. Je vais en donner un exemple, et je choisis pour cela un de leurs plus beaux airs appelé Liuye jin. Je vais noter la partition. " Notes, signes musicaux, partition, les termes sont présentés clairement, et Rousseau s'avère impardonnable. Dès 1780, un auteur français (La Borde 1780 : T. I, 143-144) qui a bien lu Amiot lui en fait le reproche : 


\begin{abstract}
« Rousseau, dans son Dictionnaire de Musique (1768), n'a pas craint d'assurer, au mot caractères, que ni les Arabes, ni les Chinois, n'avaient point de caractères pour noter les sons. Nous avons en main de quoi détruire une assertion aussi hardie. On verra, ci-après, au Chapitre de la Musique des Arabes, comment ils notent leurs airs. Quant aux caractères musicaux des Chinois, il n'est pas de notre objet de donner ici les divers exemples qu'on en trouve dans les planches qui accompagnent le Mémoire $\mathrm{du}$ P. Amiot; mais nous allons rapporter une partie des détails où ce savant Missionnaire est entré à cet égard, dans les manuscrits qu'il avait adressés en 1754 à M. de Bougainville (cahier C, pag. 53 \& suivantes) ; \& nous présenterons en original l'air Chinois que Rousseau a donné dans son Dictionnaire. »
\end{abstract}

\title{
La feuille de saule
}

Amiot (fig. 2) reprendra plus tard et par deux fois (Amiot 1754:139; Amiot 1779: $3^{\mathrm{e}}$ cahier $n^{\circ}$ 9) l'air donné par Du Halde (1735: Tome III, 328). «La feuille de saule» connaîtra une aventure unique puisque, copiant Rousseau (1768: planche N), il sera utilisé par Carl Maria von Weber (1786-1826) dans son Ouverture chinoise (perdue) puis dans sa Turandot (1809). Ce thème, en particulier la présence du fa dans le pentatonique ré-mi-sol-la-si, une erreur de Rousseau (loc. cit.) ${ }^{3}$, inspirera Paul Hindemith (1895-1963) dans le deuxième mouvement de ses Weber Metamorphoses (1943).

Fig. 2 : Notation gongche écrite verticalement et sa traduction sur portée écrite horizontalement, " La feuille de saule » (Amiot $1754:$ 139)

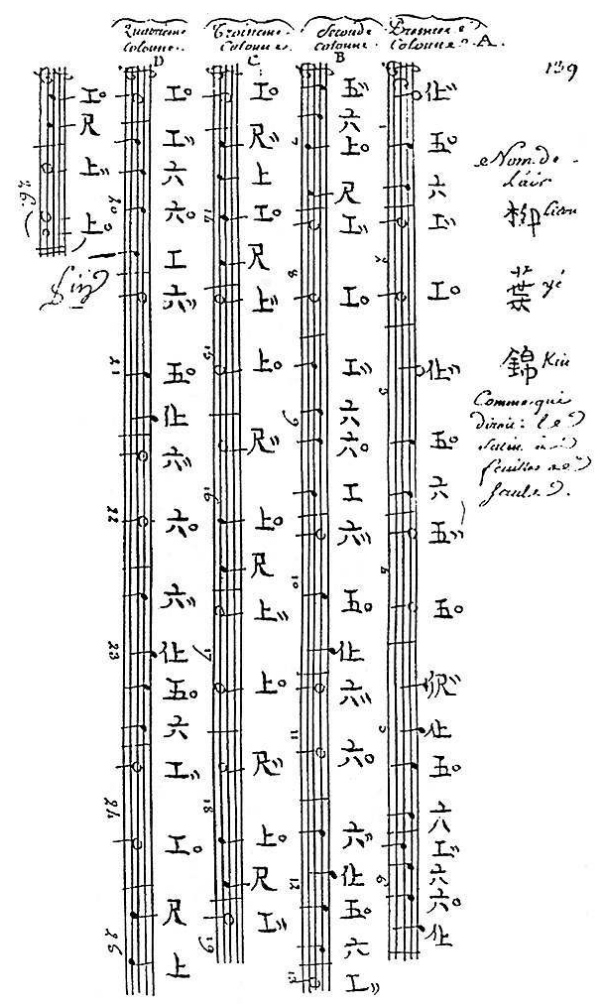

Les ronds vides marquent les premiers temps, les " marquent les seconds temps de la mesure. Les mesures sont numérotées de 1 à 26 par Amiot

En Chine, cet air connaît de très nombreuses versions encore vivantes. Tant la version d'Amiot que celle donnée par Du Halde concordent tout à fait avec celles de la région du Bas-Yangtse (Sun 1965 : 48) ou des Na-khi (Yang 1996), plus de deux siècles après. 
Les notations gongche posent un problème de traduction dû à la difficulté à déterminer la hauteur exacte des notes si et fa, qui varient dans la pratique selon le tempérament des instruments (elles sont souvent "neutres ", partageant l'intervalle de tierce mineure en deux intervalles d'une valeur de trois-quarts de ton) et selon les genres. Amiot écrit en do majeur (sans armature à la clé) mais en système sol-la-si-ré-mi ce que les Chinois modernes et les auteurs instruits à leur école comme moi traduisons unanimement en système do-ré-mi-sol-la ${ }^{4}$. Pour moi, la traduction d'Amiot serait tout à fait exacte avec un fa\# à la clé, même s'il n'est jamais joué ! On notera l'exact caractère de traduction de la double notation d'Amiot, puisque tous les signes du système gongche ont leur équivalent sur la portée.

11 Nous proposons (fig. 3) une comparaison de la version donnée par Amiot (1754), quasiment identique à Du Halde (1735) et Amiot (1779), et de la version traditionnelle (Yang 1996) de l'ensemble de musique ancienne des Na-khi de Dayan, à Lijiang, au Yunnan, intitulée bizarrement Shui long yin (Imploration du dragon d'eau). Cette dernière met en évidence l'usage de la traduction dans la tradition chinoise. Elle reproduit en effet en parallèle la notation gongche classique, selon le maître He Yi'an, et sa traduction en notation chiffrée Chevé par Yang Zenglie, musicologue et musicien du groupe. Ces indications de source figurent clairement en haut et à droite de la partition, en regard du titre et du genre. Figurent également la clé ( $\mathrm{C \# )}$ et le tempo (noire à 70), ainsi que les barres de reprise. Ajoutons qu'ici la notation gongche omet la différenciation entre les octaves médium et aiguë. Un point très remarquable de la traduction de Yang Zenglie est qu'il précise la valeur effective du signe shang (do) qui dans ce genre particulier peut représenter deux notes, si ou do, qu'il écrit donc 7 ou 1. Sous cette double notation, j'ai transcrit l'air donné par Amiot, en faisant une partition d'analyse. 
Fig. 3 : « Imploration du dragon d'eau », Yang Zenglie, ms. inédit, 1996

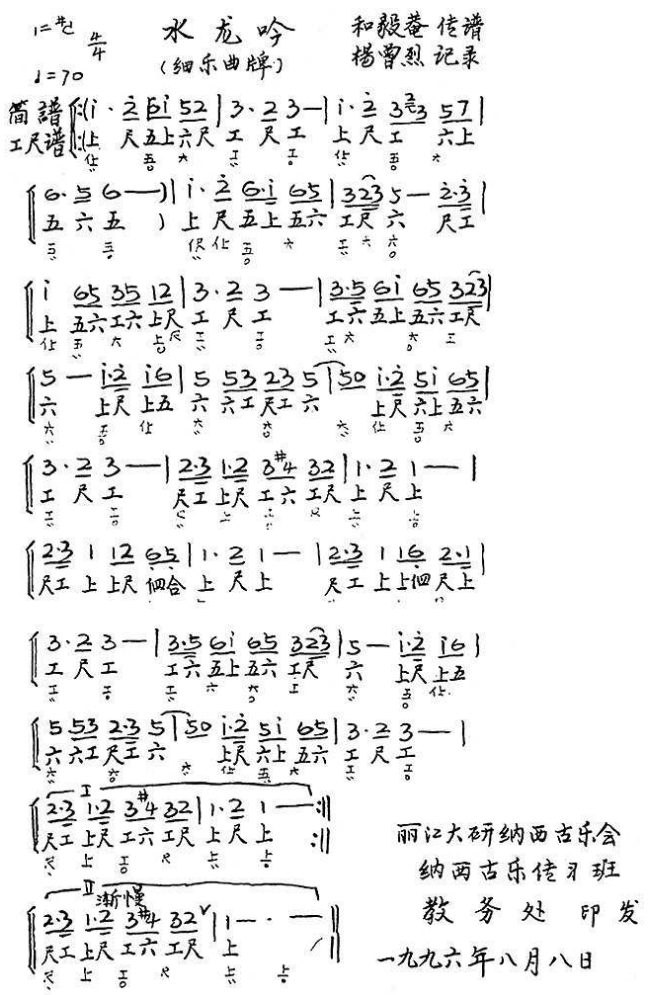

Première ligne : notation chiffrée par Yang Zenglie. Deuxième ligne : notation gongche d'après He Yi'an. Troisième ligne : report par F. Picard de la notation gongche d'Amiot (1754 : 139)

\section{Une notation de jeu}

On débattera certainement dans ce dossier comme on l'a trop fait ailleurs du caractère prescriptif ou descriptif, voire normatif, des notations musicales. Les musiciens chinois, qui ne lisent ni le tchèque, ni l'anglo-américain, ni le français, savent très bien à quoi s'en tenir. Maître Xu Chaoming, professeur d'orgue à bouche au conservatoire de musique de Shanghai et musicien hors pair, participait en janvier 1999 à des séances d'enregistrement à Paris. A son arrivée à Paris, je le prévins qu'il allait avoir à jouer, outre la partie de flûte de «Pierre et le loup» (op. 67, 1936) de Serge Prokofiev (1891-1953) au programme, un solo traditionnel ayant rapport avec l'oiseau que son instrument y personnifiait. Je lui proposai d'interpréter une très belle pièce de sa région, « Le vol de la perdrix » (Zhegu fei), et lui fournis des partitions de diverses natures : l'air de base (fig. 4) noté en gongche et en chiffré par Zhuang Naihe (1926: 79) (aide-mémoire ?), une version pour flûte solo (fig. 5) d'un air appelé « La Longévité » (Wanshou wujiang) apprise lorsque j'étudiais au CEMO et retranscrite de ma main (prescriptive ?), et d'autres versions telles que celles courantes de l'interprétation historique de Lu Chunling (1960), notée à la triple croche près, y compris la célèbre faute de mémoire qui l'affecta lors de l'enregistrement, transcription (descriptive?) d'une interprétation particulière devenue normative par l'impression et la diffusion dans les manuels. 
Fig. 4 : Notations Chevé et gongche, « Le vol de la perdrix » (Zhuang 1926)

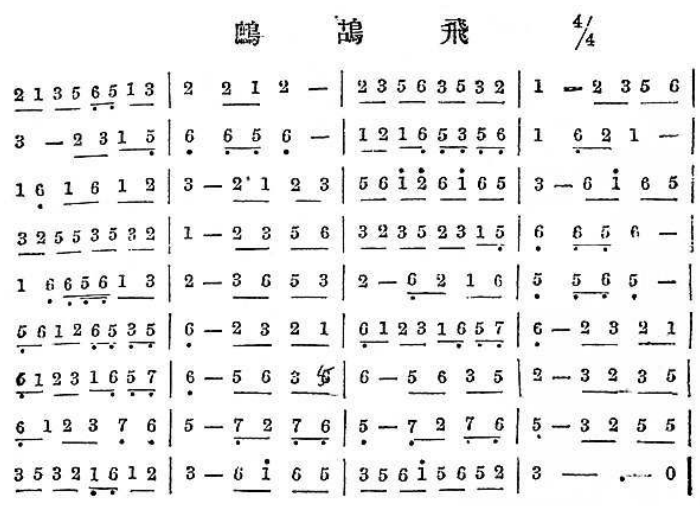

Deux erreurs ( 5 soit sol, mesure 26 de la notation chiffrée et si, la grave, au lieu de he, sol grave, dans la notation gongche) ont été rectifiées manuellement par François Picard. La partie inférieure indique l'origine de l'air, province du Hunan, les tonalités (xiao gong diao pour la flûte xiao, corde intérieure che [ré] et corde extérieure he [sol grave] pour la vièle erhu). En fin d'air, la mention « Pour cet air, on n'emploiera pas la flûte traversière dizi, mais de préférence un instrument grave pour exprimer le mouvement calme et élégant. » 
Fig. 5 : Notation Chevé, « Le vol de la perdrix », version CEMO, 1983, collection F. Picard

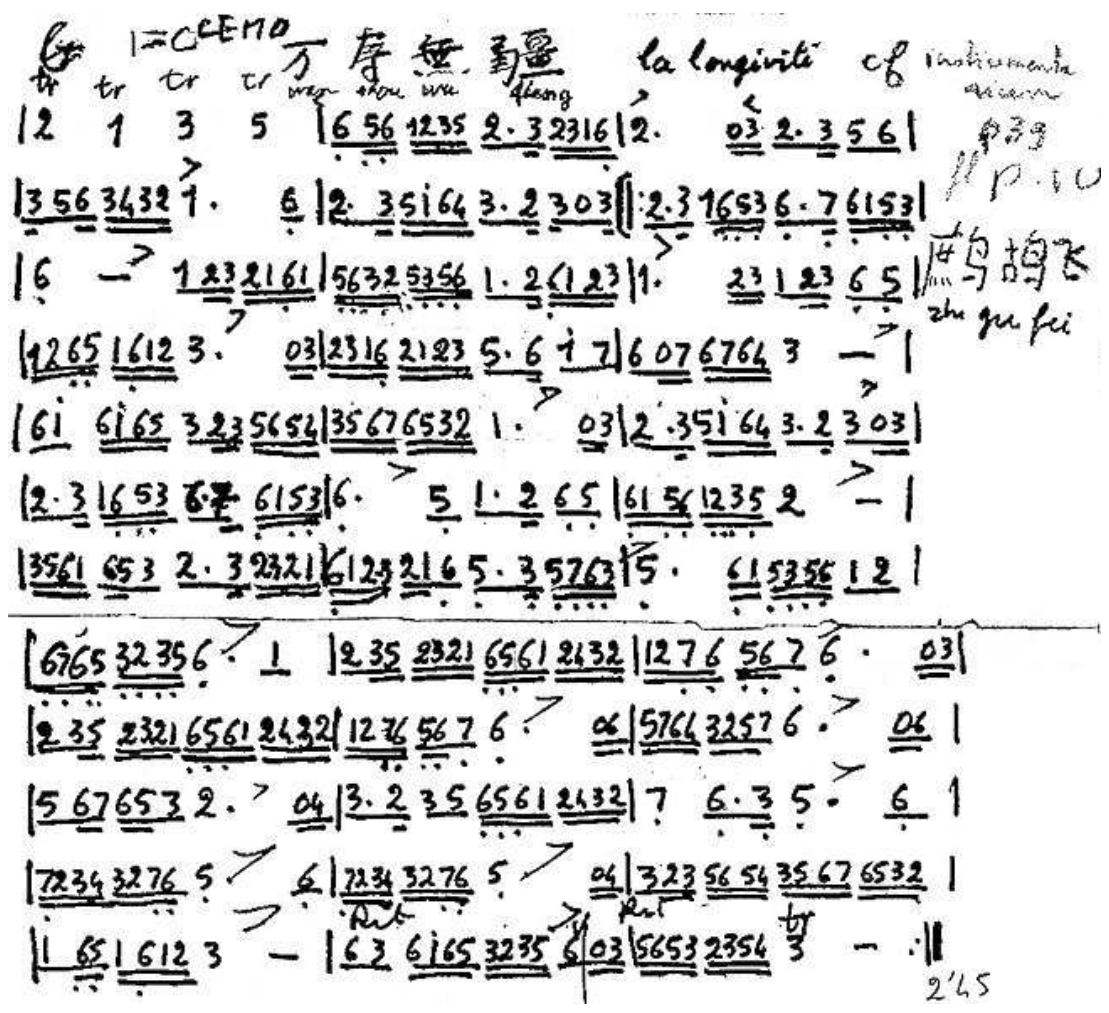

13 Maître $\mathrm{Xu}$ écouta, discuta, regarda, lut, joua, travailla, écrivit, joua, et aboutit à une notation qui correspondait exactement à ce qu'il avait besoin de noter pour lui-même afin de jouer lors de l'enregistrement. Il écrivit sous le titre, au centre et entre parenthèse, " Notation de jeu pour orgue à bouche solo » (Sheng duzou yanzou pu), et en bas à droite le lieu et la date. Cette partition (fig. 6) eut enfin un autre usage, celui de support pour noter les parties, phrases ou notes à reprendre lors de l'enregistrement (description puis prescription) puis de guider le montage (prescription normative). 
Fig. 6 : Notation Chevé, « Le vol de la perdrix », version autographe de Xu Chaoming, 1999, collection F. Picard

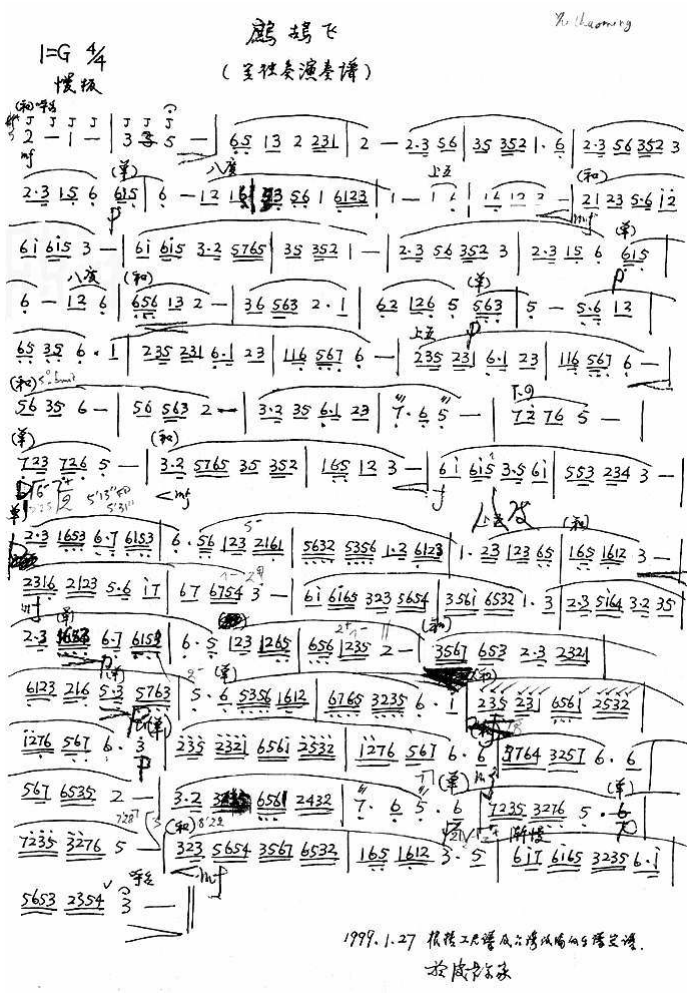

\section{L'usage et la valeur sociale des notations chiffrées}

Comment sont produites les notations de type gongche? Le musicien apprend d'oreille à chanter l'air. Puis il chante le nom des notes. Quittant le groupe ou le maitre, il transcrit le nom des notes sur papier. On peut bien sûr recopier une partition, mais là n'est pas la démarche traditionnelle. Quoi qu'il en soit, la référence à l'oralité est évidente, constante. Il en est exactement de même dans l'utilisation de la notation chiffrée (Chevé) chez les musiciens chinois. J'ai ainsi entendu des habitants de Shanghai ou du Fujian ne parlant pas d'autre langue que leur langue locale chanter « do lei mi fa so la hsi ». Jamais on ne chantera le nom des chiffres (yi er san si wu liu qi), sauf si l'on est un jeune musicien de conservatoire qui juge son élève française trop débile pour attribuer deux phonétisations au même signe ${ }^{5}$. De même les Allemands ne chantent jamais A B C D, mais la si do re. Les signes 1, 2, 3 etc. utilisés dans une partition musicale sont pour les Chinois, qui savent ce qu'est un signe graphique et une transcription phonétique, des références à des notes à travers leurs noms, et en aucun cas à des chiffres ou des nombres. Ils s'écrivent de la même façon, voilà tout, comme shang gong che etc. n'ont jamais signifié "dessus ", «travail », «pied» etc. Les deux systèmes présentent la même structure, les mêmes qualités, que l'on retrouvait dans la notation pour luth de l'époque Tang et dans toutes les nombreuses variantes (Fujian, Guangdong, Wutai shan) : les signes sont formés de peu de traits chacun, ils ne présentent pas d'ambiguïté, ils se prononcent selon des phonétisations bien différenciées et faciles à chanter. Bien sûr, les tentatives de remotiver ces signes, qui ne renvoient qu'à des notes, pour en faire des symboles, n'ont pas manqué, comme en Occident avec la solmisation. 
Fondamentalement heptatonique, la notation gongche contredit le dogme du caractère pentatonique de la musique chinoise, qui connaissait deux types de dénomination des hauteurs, le système lülü des douze hauteurs absolues et le système wusheng des cinq rapports de hauteur. Les douze lülü sont désignés par des mots bisyllabiques écrits avec des caractères complexes, et les " cinq sons " avec également des caractères formés de nombreux traits, donc longs à écrire et à lire. De plus ces deux systèmes d'écriture font référence à tout un système dogmatique. Comment exprimer et écrire une musique qui ne se limite pas à ce système et qui n'est pas pensée dans ce système? En inventant un système autonome, composé de signes nouveaux et ne renvoyant qu'à la pratique du musicien. C'est ce qu'on voit à l'œuvre dès les premières notations, les tablatures pour flûte (Annen 880) ou pour luth (Dunhuang 933). Les partitions de musique (et non les simples explications théoriques) écrites en notation lülü ou wusheng sont extrêmement rares et limitées aux musiques strictement confucéennes (Zhu 1605) (Shijing 1788) (Wenmiao 1845), où elles se sont imposées pour des raisons idéologiques évidentes. Les tablatures pour cithare qin elles-mêmes (Liebermann 1983) et pour des raisons pragmatiques semblables sont indépendantes de ces notations, sauf en ce qui concerne la notion de hauteur absolue.

\section{Retour en Europe}

Nous allons maintenant tenter d'utiliser les acquis de l'imprégnation ethnomusicologique et de la réflexion qui s'ensuivit pour relire l'histoire de la notation chiffrée en Europe.

La notation chiffrée élaborée par Rousseau a été publiée en 1742 et 1746 . On s'est demandé s'il n'avait pas été influencé par Du Halde (1735). Mais Rousseau semble avoir eu une trop piètre connaissance de l'art de lire et de citer les textes et les explications de Du Halde étaient par trop fragmentaires. Laissons-lui le mérite de cette invention qu'il importe de situer dans son contexte, celui de la démocratisation et de la pédagogie, bien dans l'esprit des Lumières. Il a voulu constituer un système de signes facile à écrire et à mémoriser, non ésotérique et qui corresponde bien à la pratique de solmisation (Rousseau 1749 : planche IV, fig. 4-7).

En fait, il est possible de trouver en Europe même des prédécesseurs de Rousseau dans une notation chiffrée pour orgue (fig. 7) exposée en français par Trichet (1640:47) :

«A ceux qui ne veulent pas prendre la peine de s'exercer aux principes de musique, l'on a accoutumé en France et en beaucoup d'autres pays de donner la tablature par lettres, dont Képler (1. 3, harmon., c. 9) parle ainsi: Sunt etiam diagrammata pro organistis fieri solita, in quibus pro lineis nigris sunt series litteratu indicantium quaenam claves tangi debeant, ubi litterae sunt pro notis, quas diagrammata cantoria in lineas aut intervalla positas adhibebant. Sur cette sorte de tablature on marque la mesure qu'on doit observer par notes de musique au-dessus de chaque réglet, de même façon qu'à la tablature du luth. Quant à la tablature d'orgue qu'on baille en Espagne par chiffres [...] comme en la musique vocale on se sert de six voix, qui sont ut, ré, mi, $\mathrm{fa}$, sol, la, aussi en cette espèce de tablature on se sert de six chiffres qui répondent à ces six voix, qui sont $1,2,3,4,5,6$, auxquelles on ajoute le 7 pour désigner une note par-dessus le la. Faisant commencer la gamme de l'orgue en la clef de $\mathrm{F}$ ut, fa, (1) signifie $\mathrm{F}$ ut, fa, $2 \mathrm{G}$ ré, sol, ut, $3 \mathrm{~A} \mathrm{mi}$, la, ré, $4 \mathrm{~B}$ fa, mi, $5 \mathrm{C}$ sol, ut, fa, $6 \mathrm{D}$ la, ré, sol et $7 \mathrm{E} \mathrm{mi}, \mathrm{la}[. .$.$] Ce qui se pourra mieux comprendre par l'exemple suivant à quatre$ parties que j'ai tiré des œuvres de musique d'Antoine Cabeçon, imprimées à Madrid, 1578 (Suit le: Kyrie du premier ton.)» 
Fig. 7 : Tablature espagnole pour orgue, Cabezón 1578 (Trichet $1640: 72-73$ )

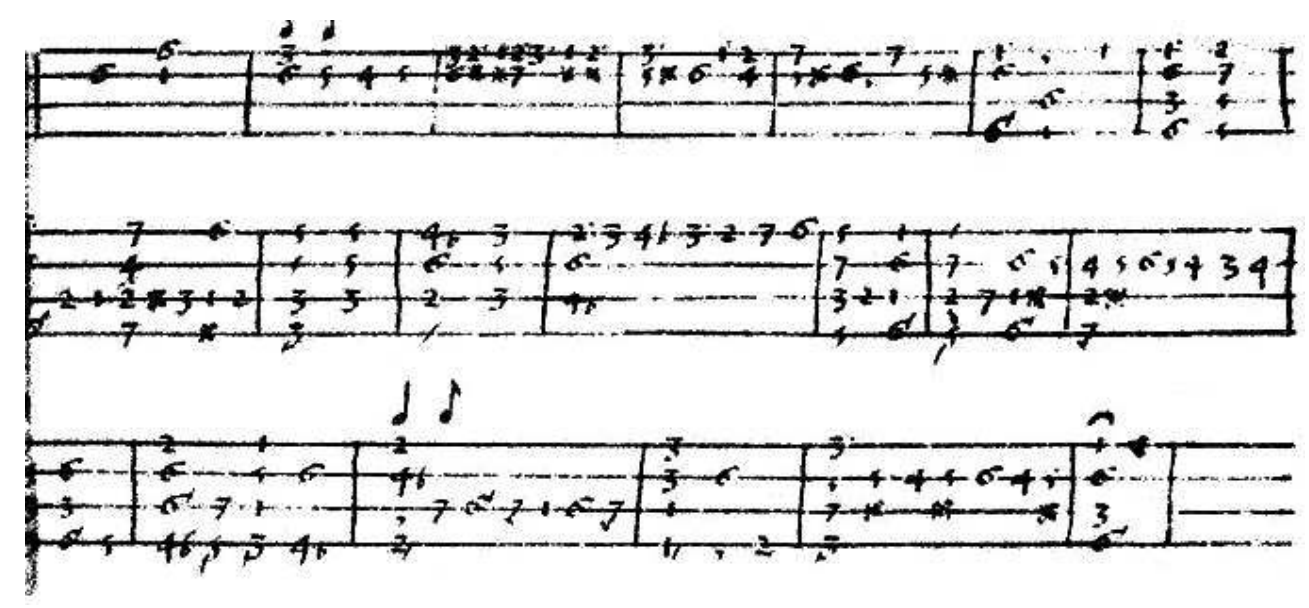

Trichet exprime à merveille la motivation des usagers de ces notations, à savoir «ceux qui ne veulent pas prendre la peine de s'exercer aux principes de musique». On remarquera qu'à cette époque on en est encore au système de solmisation complexe par hexacordes $\mathrm{F}$ ut, fa, etc. lié à l'absence du «si » pour désigner cette «note par-dessus le la ». Non seulement le solfège de l'époque avec ses notes et ses lignes était difficile à écrire, à lire et fort coûteux à imprimer, mais il était de surcroît difficile à chanter. On comprend aisément dans ces conditions combien l'envie d'un système simple se faisait sentir, attestée par tant de « solfèges faciles » et de « nouvelles méthodes pour apprendre en peu de temps".

Le système espagnol de tablature pour orgue fut décrit pour la première fois par Juan Bermudo (c. 1510 - c. 1565) en 1555 et utilisé par L. Venegas de Henestrosa en 1557. Hernando de Cabezón (1541-1602) l'utilise pour la notation des musiques de son père Antonio (1510-1566).

Quant aux successeurs de Rousseau, ce sont les pédagogues Pierre Galin (1786-1821) et Aimé Paris et Emile Chevé (1804-1864). Au début du xx siècle, les Japonais l'introduisent en Chine où elle prend le nom de «notation simplifiée » (jianpu) et s'impose rapidement, se substituant trait pour trait aux anciennes notations de type gongche. Elle a quasiment disparu en Europe, mais ses dernières traces sont vivantes dans la mémoire des musiciens populaires. Ainsi Bernard Fourtet, joueur de serpent et membre du prestigieux ensemble des Sacqueboutiers de Toulouse, l'appelle par tradition orale « solfège de Rousseau »; il a hérité par famille de partitions pour mandoline (la Toulousaine, la Marseillaise...) et d'autres pour orphéon (fig. 8) ayant en particulier appartenu à l'Orphéon « la Cigale toulousaine » fondé en 1894. On relève des musiques telles que «Les Cosaques », paroles de Paul Réal, musique d'Emile Magnin, le "Chœur de Soldats (de Sarah)», opéra de Grisar, ou encore « Nocturne », paroles de H. de Vos, musique de Camille de Vos, « imposé en Seconde Division au Concours musical de Pau (30 et 31 mai 1897)». On notera l'appellation "Ton de Ré» "Ton de Mi», "Ton de Sol», et les indications métronomiques. Les altérations sont marquées par barrage oblique de la note. 
Fig. 8 : Notation Chevé, Orphéon « la Cigale toulousaine », c. 1900, collection B. Fourtet

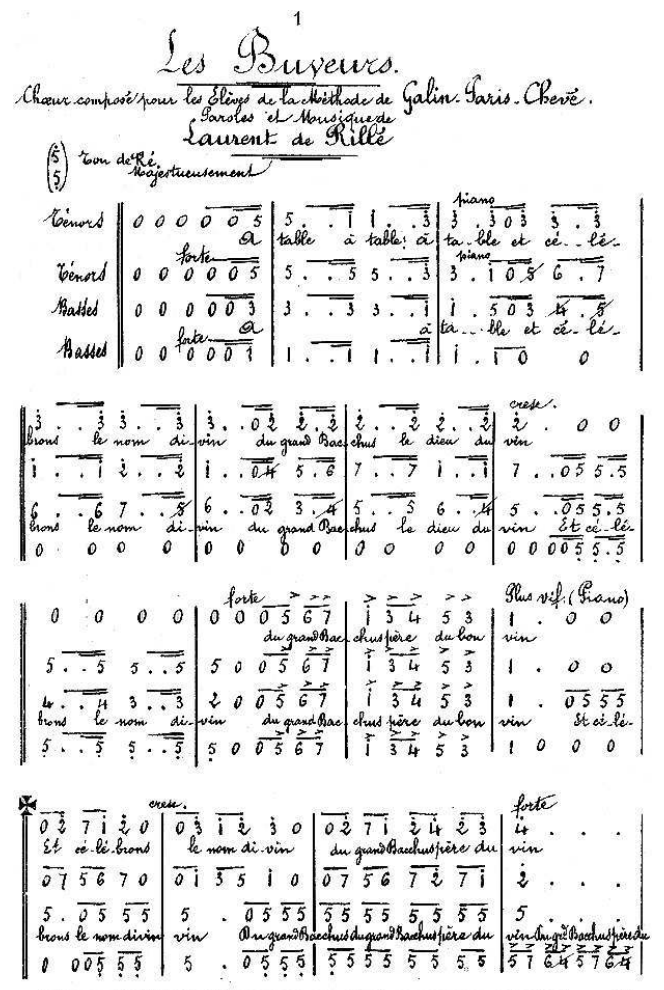

22 Moins d'un siècle plus tard, la notation chiffrée avait disparu des mémoires de la plupart des musiciens populaires, qui restaient néanmoins réfractaires à la normalisation scolaire imposée par le solfège. Les fiches (fig. 9) de la fanfare Eustache et ses trompes, active à Gennevilliers c. 1980, témoignent de la nécessité d'une notation facile à apprendre, rapide à écrire, lisible, maniable, concise, et de la référence absolue que constitue le nom des notes, leur vocalisation. Tout simplement, sur les fiches de bristol que porteront en extérieur les lyres, on inscrit le nom des notes en toutes lettres. 
Fig. 9 : Notation en toutes lettres, « $81 / 2$ », fanfare Eustache et ses trompes, c. 1980, collection F. Picard

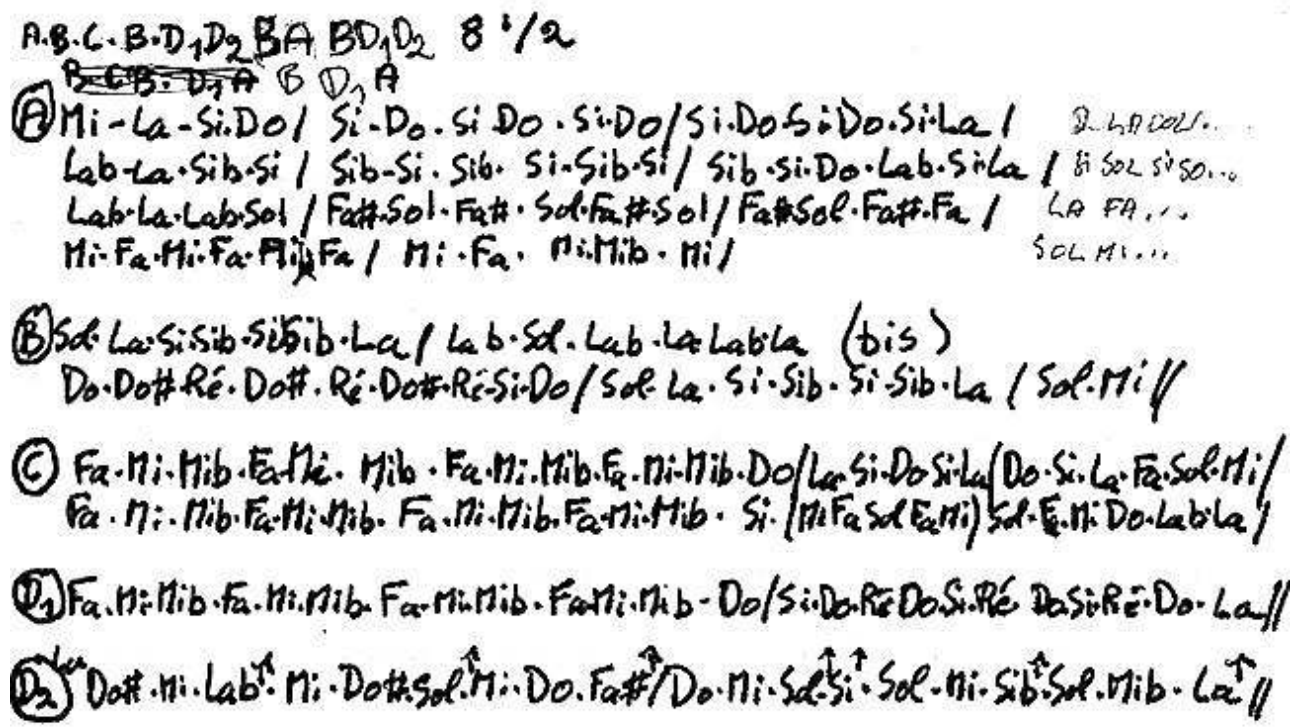

On remarquera que seules figurent les valeurs de hauteur, pas même les durées, l'octave étant parfois indiquée par des flèches vers le haut ou vers le bas.

La forme est indiquée par des lettres (A.B.C...), mais un chiffrage d'accord peut coexister (tel A7 A7 Dm Dm A7 A7 Dm Dm), non sans ambiguïté. A la même époque, la fanfare des Beaux-Arts tentait de forcer cette notation à évoluer, on inventait des signes pour les durées, tandis qu'en Colombie on écrivait aussi le nom des notes en toutes lettres « mi » «f $»^{6}$.

Je ne saurais conclure que par le souhait que ce modeste article contribue à une réintroduction de la méthode Rousseau-Chevé dans l'enseignement et les pratiques collectives en Europe, et sous la forme unifiée que connaissent et pratiquent quelques centaines de millions de Chinois de République Populaire ou de Taïwan. J'ajouterai une anecdote empruntée à une tierce culture (Van Zanten 1995). Le Java Instituut souhaitait promouvoir une méthode de notation unique et unifiée. Il organisa en 1921 un concours où furent représentées des notations chiffrées, des représentations graphiques des touches des instruments et la notation sur portée. Cette dernière, jugée plus scientifique, fut retenue et décrétée obligatoire. Quelques années plus tard, la pratique universelle, telle aussi que j'ai pu l'apprendre au sein de l'atelier de gamelan du Musée de l'Homme, s'en tenait à la notation chiffrée, qui plus est défective, puisque 12356 faisaient référence aux notes do-ré-mi-sol-la du pentatonique auquel on identifiait l'échelle des hauteurs et non à une simple numérotation des touches, d'ailleurs limitées à cinq.

\section{Oralité, geste, image et notation}

La notation musicale, plus qu'un dessin, un graphisme, est une écriture. Elle en présente beaucoup de caractéristiques: système de signes, arbitraire du codage, référence à la langue. 


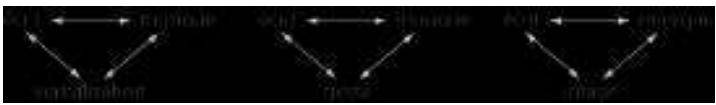

Dans ces trois tableaux, le sens des flèches n'est pas donné une fois pour toutes. La médiation verbale me semble la plus courante, pour ne pas dire la plus naturelle, celle du geste correspond à ce que l'on appelle par convention "tablature » (luth Renaissance, guitare folk, cithare qin...), celle par l'image est exposée par Arbaretaz (1979: 6), «Un intervalle est toujours représenté sur la portée par la même distance. Il est donc possible de « VOIR » directement un intervalle, sans avoir besoin de compter ». Cette auteur met ainsi en valeur un des avantages de la notation sur portée, son caractère visuel, alors que son caractère graphique ne me semble pas un avantage prépondérant: difficultés d'écriture et d'impression, d'apprentissage du codage. Pour celui qui a appris à suivre au tempo une partition d'orchestre, ce caractère visuel manque dans les notations chiffrées, si linéaires. Les notations proprement graphiques du Tibet (Helffer 1994) ou du chant taoïste (Yuyin fashi 1444), si expressives soient-elles, manquent du caractère discret, reproductible, dicible qui en ferait une écriture.

\section{BIBLIOGRAPHIE}

AMIOT Joseph-Marie, 1754, De la Musique moderne des Chinois (An., ms., s.l., s.d.), Pékin, Paris, Bibliothèque nationale de France.

AMIOT Joseph-Marie, 1779?, Divertissements chinois ou Concerts de musique chinoise, ms., Pékin. Paris : Bibliothèque nationale de France. I « Les notes chinoises mises sur des lignes, à notre manière. » et II « Les notes purement à la chinoise. »

ANNEN, 880, Shittan zô (Canon du siddham), rééd. Taishô Tripitaka, vol. LXXXIV, n 2702.

ARBARETAZ Marie Claude, 1979, Lire la musique par la connaissance des intervalles. Paris : Chappell.

BERMUDO Juan, 1555, Declaración de instrumentos musicales. Ossuna (rééd. S. Kastner, ed., Kassel : Bärenreiter, 1957).

CHAO Rulan Pian, 1969, Sonq Dynasty Musical Sources and their Interpretation. Cambridge, Mass.: Harvard University Press.

COURANT Maurice, 1921, « Histoire de la musique- Chine et Corée », dans Lavignac, Encyclopédie de la musique et Dictionnaire du Conservatoire, vol. 1. Paris : Delagrave : 95-96.

DU HALDE Jean-Baptiste, 1735, Description géographique, historique, chronologique, politique, et physique de l'Empire de la Chine et de la Tartarie chinoise. Paris : P.G. Le Mercier.

DUNHUANG, 933, Manuscrit de Dunhuang, BNF, fonds Pelliot chinois P. 3808 [avant 933].

HELFFER Mireille, 1994, Mchod-rol, les instruments de la musique tibétaine. Paris : CNRS éditions, Editions de la Maison des Sciences de l'Homme. (collection Chemins de l'ethnologie).

JAO Tsong-Yi, 1971, Airs de Touen-Houang. Trad. Paul Demiéville. Paris : CNRS. 
JI Liankang, 1986, Sui Tang Wudai yinyue shi liao (Matériaux pour l'histoire de la musique sous les Sui, les Tang et les Cinq Dynasties). Shanghai : Wenyi.

LA BORDE Jean-Benjamin de, 1780, Essai sur la musique ancienne et moderne. Paris : Ph.-D. Pierres (4 vol. ).

LALOY Louis, 1912, La Musique chinoise : étude critique. Paris : Henri Laurens.

MA Hiao-Tsiun, 1976, « Chine », Marc Honegger, ed. : Science de la musique. Paris: Bordas, vol. A$\mathrm{K}$.

PERNY Paul, M.A., 1872, « Appendice n XIV. Musique des Chinois », Appendice du Dictionnaire français-latin-chinois de la langue mandarine parlée. Paris : Firmin Didot.

PICARD François, 1991, La musique chinoise. Paris : Minerve.

PICARD François, 1999, « Les notations musicales en Chine », Hugues Genevois et Yann Orlarey, ed. : Musique \& Notations. Aleas, collection Musique \& Sciences.

ROUSSEAU Jean-Jacques, 1742, « Projet concernant de nouveaux signes pour la musique ». Paris : Académie des Sciences, 22 août.

ROUSSEAU Jean-Jacques, 1746, « Dissertation sur la musique », repris in : Ecrits sur la musique. Paris : Stock, 1979.

ROUSSEAU Jean-Jacques, 1749, « Musique », in : Denis Diderot et d'Alembert, Encyclopédie, Paris. Rousseau Jean-Jacques, 1768, Dictionnaire de Musique. Paris: Duchesne. (Rééd. Hildesheim / New York: Georg Olms / Johnson, 1969).

SHEN Gua, 1095, Mengqi bitan et Bu bitan, 1086-1095, rééd. Hu Daojing, éd., Mengqi bitan jiaozheng, Zhonghua shuju, 1962. Hu Daojing, éd., Yuan kan Mengqi bitan (fac simile d'une édition postérieure à 1305). Pékin : Wenwu, 1975.

SHIJING, 1788, [An.] Shijing yuepu (Notations musicales du Classique des poèmes)(Rééd. Shanghai, 1937).

SOULIE Georges , 1911, La Musique en Chine, (extrait du Bulletin de l'Association Franco-Chinoise). Paris : Ernest Leroux.

STAAL Fritz, 1990, Jouer avec le feu, Pratique et théorie du rituel védique. Paris : Collège de France, Institut des civilisations indiennes.

SUN Yude, 1965, Dongxiao chuizou fa (Méthode de flûte droite) (Rééd. Hong Kong : Xianggang shuju).

TRAN Van Khê, 1990, « Musicales (traditions) - Musiques d'inspiration chinoise ». Paris :

Encyclopcedia Universalis, vol. 15.

TRICHET Pierre , 1640, Traité des instruments de musique, rééd. François Lesure, ed. Paris, 1957 ; Genève : Minkoff, 1978.

Van Aalst J.A., 1884, Chinese Music, 1884, Shanghai: Imperial Customs Series, vol. 6 (Rééd., Pékin: French Bookstore, 1933. Rééd. New York: Paragon, 1964).

VAN ZANTEN Wim, 1995, « Notation of music; theory and practice in West Java ». Oideion. The performing arts worldwide 2: 209-233.

Venegas L. de Henestrosa, 1557, Cifra nueva para tecla, arpa y vihuela, Alcalá de Henares. (Rééd. H. Anglès, ed., Monumentos de la Música Española, II, Madrid et Barcelone, 1944). 
WANG Pin Lu, Liebermann Frederic (trad.), 1983, A Chinese Zither Tutor, The Mei'an ch'in pû. Seattle \& London: University of Washington Press.

WENMIAO, 1845, [An.] Wenmiao dingli pu (Partitions des rites du temple de la Littérature). Hunan, 1845, (Rééd. Shanghai: Xuesa saoju, 1890).

WIDDESS Richard, 1995, The Ragas of Early Indian Music: Modes, Melodies and Musical Notations from the Gupta Period to C. 1250. Oxford : Clarendon Press.

YANG Zenglie, 1996, Shuilong yin (Imploration du dragon d'eau), ms. inédit.

Yuyin Fashi, 1444, [An.] Yuyin fashi (Rituel des sons de jade), juan 1, section 2. Daozang (Canon taoïste), numéro 333.

ZHU Zaiyu, 1605, Caoman guyue pu (Partitions de musique ancienne de Caoman).

ZHUANG Naihe, 1926, Zhongguo yayue ji (Anthologie de musique classique chinoise). Shanghai.

\section{Références discographiques}

FRISCH Jean-Christophe, 1996, Joseph-Marie Amiot, «Liéou yé kin Pièce de soie à l'imitation des feuilles de saule ", 1754, Jean-Christophe Frisch, traverso, Teodorico Pedrini : Concert baroque à la cité interdite, XVIII-21 Musique des Lumières. CD Astrée Auvidis E 8609, pl. 27.

HE Yi'an, s.d., « Shui long yin (Imploration du dragon d'eau) », ensemble de musique ancienne des Na-khi de Dayan, Lijiang, Yunnan, cassette Naxi guyue di yi ji - Lijiang Dongjing yinyue (Musique ancienne des Na-khi, vol. 1, Musique du Canon des grottes de Lijiang), Yunnan Yinxiang, face B plage 2 .

LU Chunlinga « Zhegu fei (Le vol de la perdrix) ", Lu Chunling, flûte traversière dizi, disque $33 \mathrm{t}$. Zhongguo changpian 3.1246, rééd. CD Ocora Chine musique classique, C 559039, pl. 5. Transcription publiée dans Conservatoire de musique de Chine, Conservatoire Central de musique, Minzu yueqi chuantong duzou qu xuanji (Anthologie des solos intrumentaux de musique traditionnelle chinoise), Pékin : Renmin yinyue, 1978, rééd. 1983 : 10-11.

PIMPANEAU Jacques, 1988, Chine, musique classique. CD Ocora C 559039.

SUN Yude, 1961, «Liu yao jin (Le saule argenté) », Sun Yude, flûte droite xiao. CD Ocora Chine musique classique, C 559039, pl. 13.

XU Chaoming, 1999, «Le vol de la perdrix » (Zhegu fei), complément à Serge Prokofiev, « Pierre et le loup », CD Virgin EMI, à paraître.

\section{NOTES}

1. Nous disons « les notations « car il en existe différentes variantes régionales ou historiques graphiques et sonores, toutes équivalentes par l'opération de traduction.

2. A propos de la notation indienne, et particulièrement l'inscription de Kudumiyamalai datant des VII ${ }^{\mathrm{e}}$-VIII ${ }^{\mathrm{e}}$ siècles, voir Widdess (1995: 102-104).

3. Cette erreur est rectifiée par Rousseau (1749 : planche III, fig. 4).

4. Les discussions menées avec André-Marie Despringre (sol-la-si-ré-mi, comme Brăiloiu) et Gilles Léothaud (fa-sol-la-do-ré, comme Tràn Van Khê) au sein du groupe de recherches en ethnomusicologie de Paris IV-Sorbonne ont mis en évidence la diversité des pratiques des 
professionnels français. L'argument d'autorité ne pouvant plus trancher, on recommandera de préciser éventuellement la valeur des pyen.

5. Sabine Trébinjac, communication orale au séminaire du Musée de l'Homme, Paris, vers 1990.

6. Marcela Gonzalez, communication à mon cours « Oralité et notations », Université Paris IV, 11 décembre 1998.

\section{RÉSUMÉS}

Pour reprendre la distinction que Frits Staal (Staal 1990 : 16-17), s'inspirant de la linguistique, propose d'appliquer à l'étude du rituel entre phonologie, syntaxe, sémantique et pragmatique, cette étude des notations insiste sur ce dernier domaine. Elle expose des notations qui font référence à une oralité, aux noms des notes, plutôt qu'à une analogie graphique. Puis elle examine les procédés de passage d'une notation à une autre, qui s'avèrent présenter les caractères d'une traduction. Les exemples sont tirés de la Chine et de l'Occident, mais aussi des passages d'une civilisation à une autre. Les relations entre musiques orales et écrites sont en outre revisitées au profit des valeurs, mises en lumière par l'herméneutique, de la transmission et de l'interprétation.

\section{AUTEUR}

\section{FRANÇOIS PICARD}

François Picard, né une année du cheval de feu à Paris, est professeur d'ethnomusicologie à l'Université Paris IV Sorbonne. Musicien, il a joué avec la fanfare Eustache et ses trompes, l'ensemble de musique médiévale et Renaissance La Maurache, le groupe de jazz-rock E. Coli, au gamelan du Musée de l'Homme. Lors de son séjour à Shanghai en 1986-87, il pratique au sein des ensembles de musique locale Jiangnan sizhu. Depuis son retour, il joue de la musique chinoise (vents) au sein de l'ensemble Fleuve Jaune puis fonde son ensemble, Fleur de Prunus, et accompagne occasionnellement les maîtres de passage. Il mène parallèlement une activité de directeur artistique. Il travaille depuis 1995 avec Jean-Christophe Frisch sur les échanges musicaux entre Européens et Chinois à Pékin aux XVII ${ }^{\mathrm{e}}$-XVIII ${ }^{\mathrm{e}}$ siècles. 